Canad. Math. Bull. Vol. 21 (2), 1978

\title{
FIXED POINTS CAN CHARACTERISE CURVES
}

\author{
BY \\ HELGA SCHIRMER
}

\begin{abstract}
The concept of a firm fixed point of a selfmap of a metric space is introduced. Loosely speaking a fixed point is firm if it cannot be moved to a point nearby with the help of a map which is arbitrarily close to the given map. It is shown that a continuum always admits a selfmap with a firm fixed point if the continuum contains a triod and if the vertex of the triod has a neighbourhood which is a dendrite. This condition holds in particular for local dendrites. Hence a local dendrite is an arc or a simple closed curve if and only if it does not admit a selfmap which has a firm fixed point.
\end{abstract}

1. Introduction. Fixed point theory often employs methods from algebraic topology-especially index theory-in order to distinguish between different kinds of fixed points. But little use has been made so far of methods from general topology in the study of special stypes of fixed points. In the present paper we use such methods to introduce and apply one particular kind of fixed point, the so-called firm fixed point.

Firm fixed points are fixed points which cannot be moved to points nearby with the help of maps which are arbitrarily close to the given one. A firm fixed point $c$ of a selfmap $f$ is by definition an isolated one, i.e. it has a neighbourhood $U$ so that $c$ is the only fixed point of $f$ on $\mathrm{Cl} U$ (the closure of $U$ ). We use Fix $f$ to denote the fixed point set of the map $f: X \rightarrow X$ in the precise definition of a firm fixed point.

Definition. A fixed point $c$ of a selfmap $f$ of a metric space $(X, d)$ is firm if there exists a neighbourhood $U$ of $c$ with $\mathrm{Cl} U \cap$ Fix $f=\{c\}$ and an $\varepsilon>0$ so that every selfmap $g$ with

(i) $g(x)=f(x)$ for all $x \in X \backslash U$,

(ii) $d(f(x), g(x))<\varepsilon$ for all $x \in X$,

(iii) $\mathrm{Cl} U \cap$ Fix $g$ is a single point

has $c$ as a fixed point.

We shall study firm fixed points on certain continua. By a continuum we mean a compact, connected and metric space. An arc is a homeomorphic

Received by the editors February 15, 1977 and, in revised form, May 13, 1977. 7579).

This research was partially supported by the National Research Council of Canada (Grant A 
image of an interval, and a simple closed curve a homeomorphic image of a circle. A (simple) triod is the union of three arcs which have exactly one point, the vertex, in common. A dendrite is defined as a locally connected continuum which does not contain a simple closed curve, and has the property that every two of its points are connected by one and only one arc. A continuum is a local dendrite if each of its points has a neighbourhood which is a dendrite. A one-dimensional continuum is called a curve, and local dendrites are examples of curves. (See e.g. [1], pp. 300, 303 for details.)

Fixed points in continua which are not firm are easy to find. Examples include all fixed points of constant maps and all isolated fixed points of all selfmaps of an arc, a simple closed curve, or any other manifold. The most typical example of a firm fixed point can be obtained if a triod is mapped into itself in such a way that the vertex $v$ is kept fixed and the map is a dilation in a neighbourhood of $v$. Such a map is described in the proof of the Theorem in §2. More generally, firm fixed points can arise in curves when branchpoints are present.

The main result, the Theorem in $\$ 2$, states that a selfmap with a firm fixed point always exists on a continuum if it contains a triod whose vertex has a neighbourhood which is a dendrite. The proof is constructive and uses the partial order structure of dendrites. A characterisation of arcs and simple closed curves in the class of all local dendrites is obtained as a corollary.

I would like to thank the referee for his helpful suggestions.

2. Results. The definition of a firm fixed point is a local one, therefore proofs relating to firm fixed points are likely to be local in character. This explains the usefulness of the following simple lemma.

Lemma 1. If the point $x$ of the continuum $X$ has a neighbourhood which is a dendrite, then $x$ has a basis $\beta_{x}$ of closed neighbourhoods whose members are dendrites.

Proof. Let $N$ be a neighbourhood of $x$. We have to find a neighbourhood $B$ of $x$ which is contained in $N$ and is a dendrite.

By assumption $x$ has a closed neighbourhood $D$ which is a dendrite. Choose a neighbourhood $M$ of $x$ with $\mathrm{Cl} M \subset N$, and let $B$ be the closure of the arccomponent of $M \cap D$ in $D$ which contains $x$. As $B$ is a subcontinuum of $D$, it is a dendrite (see [1], p. 301), and as $D$ is locally connected, $B$ is a neighbourhood of $x$.

The elements $B \in \beta_{x}$ are called the basic neighbourhoods at $x$. If $x_{1}, x_{2} \in X$ are contained in a subdendrite $D$ of $X$, then we denote by $\left[x_{1}, x_{2}\right]_{D}$ the (unique) arc from $x_{1}$ to $x_{2}$ in $D$, and by $\left(x_{1}, x_{2}\right)_{D}$ the open arc $\left[x_{1}, x_{2}\right]_{D} \backslash\left\{x_{1}, x_{2}\right\}$. We interpret $[x, x]_{D}$ as the point $x$. 
We separate one more lemma from the proof of the Theorem, in order to show more clearly how the local dendritic structure enters into it.

LEMma 2. Let the point $x$ of the continuum $X$ have a neighbourhood which is a dendrite, let $f$ be a selfmap of $X$ and $B, B^{\prime}$ be basic neighbourhoods at the point $x \in X$ such that $B \subset B^{\prime}$ and $f(B) \subset B^{\prime}$. If there exist points $x_{1}, x_{2} \in B$ with $x_{1} \in\left(x_{2}, f\left(x_{1}\right)\right)_{B^{\prime}}$ and $x_{2} \in\left(x_{1}, f\left(x_{2}\right)\right)_{B^{\prime}}$, then $\left(x_{1}, x_{2}\right)_{B^{\prime}} \cap$ Fix $f \neq \phi$.

Proof. Take $f\left(x_{1}\right)$ as the root of a partial order $\leq$ of the dendrite $B^{\prime}$ which is continuous and order dense [3], [4]. The graph of the partial order is closed in $B^{\prime} \times B^{\prime}$, and therefore the set

$$
E=\left\{x \in\left[x_{1}, x_{2}\right]_{B^{\prime}} \mid x \leq f(x)\right\}
$$

is closed in $\left[x_{1}, x_{2}\right]_{B^{\prime}} . E$ is non-empty as $x_{2} \in E$, and it is $\neq\left[x_{1}, x_{2}\right]_{B^{\prime}}$ as $x_{1} \notin E$. Let $x_{0}$ be its minimum. Then $x_{0} \in E$, hence $x_{0} \leq f\left(x_{0}\right)$. If $x_{0}<f\left(x_{0}\right)$, then the continuity of $f$ implies that there exists an open subset $U$ in $\left[x_{1}, x_{2}\right]_{B^{\prime}}$ such that $x_{0} \in U$ and $x<f(x)$ for all $x \in U$, which contradicts the minimality of $x_{0}$. Hence $x_{0} \in\left(x_{1}, x_{2}\right)_{B^{\prime}} \cap$ Fix $f$.

We now state and prove the main result.

THEOREM. A continuum admits a selfmap with a firm fixed point if it contains a triod and if the vertex of this triod has a neighbourhood which is a dendrite.

Proof. Let the continuum $(X, d)$ contain a triod $T$ which is the union of three arcs $\left[c, a_{i}\right]$, where $i=1,2,3$. The triod $T$ can be given a convex metric $d_{T}$, and hence every $x \in T$ can be written in the form

$$
x=(1-\lambda) c+\lambda a_{i}, \quad 0 \leq \lambda \leq 1,
$$

where $\lambda$ is determined by

$$
d_{T}(x, c)=\lambda d_{T}\left(x, a_{i}\right)
$$

Define $f^{\prime}: T \rightarrow T$ by

$$
f^{\prime}(x)=(1+\mu) c+\mu a_{i}
$$

with

$$
\mu=\lambda+\frac{1}{2} \lambda(1-\lambda)
$$

As $T$ is contractible, there exists a retraction $r: X \rightarrow T$, so we can define $f: X \rightarrow X$ by $f=i \circ f^{\prime} \circ r$, where $i: T \rightarrow X$ is the injection. Then Fix $f=$ Fix $f^{\prime}=$ $\left\{c, a_{1}, a_{2}, a_{3}\right\}$. We show that $c$ is firm if $c$ has a neighbourhood which is a dendrite.

In order to construct $U$ and $\varepsilon$, Let $U^{\prime}$ be the open set $r^{-1}(U$ $\left.\left(\left[c, a_{i}\right)_{T} \mid i=1,2,3\right)\right)$, choose a neighbourhood basis $\beta_{c}$ of $X$ at $c$ which consists 
of dendrites, and a $B^{\prime} \in \beta_{c}$ with $B^{\prime} \subset U^{\prime}$. Select $\eta>0$ so that the $\eta$-ball

$$
U(c, \eta)=\{x \in X \mid d(x, c)<\eta\}
$$

is contained in $B^{\prime}$, and determine $\delta>0$ so that $f(U(c, \delta)) \subset U\left(c, \frac{1}{2} \eta\right)$. Let $\varepsilon=\min \left(\delta, \frac{1}{2} \eta\right)$, and $U=B$ be any basic neighbourhood with $c \in B \subset U(c, \varepsilon)$.

The firmness of $c$ will be demonstrated with the use of Lemma 2. Note that $B \subset B^{\prime}$, and also $\left.U(f(B)), \varepsilon\right) \subset B^{\prime}$, where

$$
U(f(B), \varepsilon)=\{x \in X \mid d(x, f(B))<\varepsilon\} .
$$

We next find, for $i=1,2,3$, a point $x_{i} \in\left(c, a_{i}\right)_{T} \cap B d B$ (where $B d$ denotes the boundary of $B$ in $X$ ) such that $\left[c, x_{i}\right]_{T} \subset B$. For this purpose, let

$$
F_{i}=\left\{x \in\left[c, a_{i}\right]_{T} \mid[c, x]_{T} \subset B\right\} .
$$

Then $a_{i} \notin F_{i}$ (as $a_{i} \notin B$ ), but $F_{i}$ contains a neighbourhood of $c$ in $\left[c, a_{i}\right]_{T}$ as $B$ is a neighbourhood of $c$. The set of $F_{i}$ is closed: If $x^{\prime} \in\left(X \backslash F_{i}\right) \cap\left[c, a_{i}\right]_{T}$, then $(X \backslash B) \cap\left(c, x^{\prime}\right]_{T} \neq \varnothing$. As B is closed, we can easily find a neighbourhood $V$ of $x^{\prime}$ so that $[c, y]_{T} \not \subset B$ for all $y \in V \cap\left[c, a_{i}\right]_{T}$, and hence $\left(X \backslash F_{i}\right) \cap\left[c, a_{i}\right]_{T}$ is open in $\left[c, a_{i}\right]_{T}$. The closed set $F_{i}$ has a maximum $x_{i} \in\left(c, a_{i}\right)_{T}$, and as $\left[c, x_{i}\right]_{T} \subset B$, we have $x_{i} \in B$. But $x_{i}$ is not contained in the interior of $B$ in $X$, as otherwise $x_{i} \in W \subset B$ for some open subset $W$ of $X$, and therefore $\left[c, x_{i}\right]_{T} \cap W \subset B$ in contradiction to the maximality of $x_{i}$. So $x_{i} \in B d B$.

Now let $g: X \rightarrow X$ be any map which fulfills the conditions (i), (ii) and (iii) of the definition of a firm fixed point, with $\varepsilon=\min \left(\delta, \frac{1}{2} \eta\right)$ and $U=B$. It follows from the definitions of $\varepsilon$ and $B$ that $g(B) \subset B^{\prime}$, and from $x_{i} \in B d B$ that $g\left(x_{i}\right)=f\left(x_{i}\right)$ for $i=1,2,3$. As the arc between any two points of a dendrite is unique, $\left[c, x_{i}\right)_{T} \subset B \subset B^{\prime}$ implies

$$
\left[x_{1}, x_{2}\right]_{T}=\left[x_{1}, c\right]_{T} \cup\left[c, x_{2}\right]_{T}=\left[x_{1}, x_{2}\right]_{B}=\left[x_{1}, x_{2}\right]_{B^{\prime}},
$$

and $\left[x_{1}, f\left(x_{1}\right)\right]_{T} \subset f\left(\left[c, x_{1}\right]_{T}\right) \subset f(B) \subset B^{\prime}$ implies

$$
\left[x_{2}, f\left(x_{1}\right)\right]_{T}=\left[x_{2}, x_{1}\right]_{T} \cup\left[x_{1}, f\left(x_{1}\right)\right]_{T}=\left[x_{2}, f\left(x_{1}\right)\right]_{B^{\prime}} .
$$

Hence it follows from Lemma 2 that $\left(x_{1}, x_{2}\right)_{B^{\prime}} \cap$ Fix $g \neq \varnothing$, and similarly that $\left(x_{2}, x_{3}\right)_{B^{\prime}} \cap$ Fix $g \neq \varnothing$. As $g$ has at most one fixed point on $\left(x_{1}, x_{2}\right)_{B^{\prime}} \cup$ $\left(x_{2}, x_{3}\right)_{B^{\prime}} \subset B$, we see that $c \in$ Fix $g$, and that $c$ is firm.

We conclude by considering the special case where the continuum is actually a local dendrite. It is well-known that a local dendrite is an arc or a simple closed curve if and only if it does not contain a triod. (See e.g. [2], p. 218.) Hence we can characterise arcs and simple closed curves with the help of firm fixed points.

Corollary. A local dendrite is an arc or a simple closed curve if and only if it does not admit a selfmap with a firm fixed point. 
3. Outlook. The result used in the derivation of the Corollary from the Theorem is true for a much wider class of spaces than local dendrites and holds e.g. for all locally connected continua. The proof of the Theorem, however, uses the local dendritic structure crucially in the application of Lemma 2, and I do not know how far the Theorem can be generalized. The example of the disc shows that it can hardly be extended beyond one-dimensional spaces, so the following question arises naturally: Does there exist a locally connected curve which contains a triod, but does not admit a selfmap with a firm fixed point?

A much more general, and less precise, question is: Can other special kinds of fixed points be defined and used in the characterisation of certain continua?

\section{REFERENCES}

1. K. Kuratowski, Topology vol. II, New Edition, New York and London, 1968.

2. R. L. Moore, Foundations of Point Set Theory, Revised Edition, Providence, R.I., 1962.

3. E. W. Ward, Jr., A note on dendrites and trees, Proc. Amer. Math. Soc. 5 (1954), 992-994.

4. -, A general fixed point theorem, Colloq. Math. 15 (1966), 243-251.

Carleton University

OTtawa, Canada K1S 5B6 